\title{
On Operators with Discontinuous Range
}

\section{G. V. Khromova}

Galina V. Khromova, Saratov State University, 83, Astrakhanskaya st., 410012, Saratov, Russia, KhromovAP@info.sgu.ru

With the use of operators from approximation function theory we construct integral operators with discontinuous range of values, which make it possible to obtain uniform approximations of continuous functions on the whole interval of their definition.

Key words: operator, continuous function, interval, uniform approximations.

The results have been obtained in the framework of the national tasks of the Ministry of Education and Science of the Russian Federation (project no. 1.1520.2014K).

\section{References}

1. Goncharov V. L. The theory of interpolation and approximation of functions. Moscow, GITL, 1954 (in Russian).

2. Khromova G. V. The problem of the reconstruction of functions that are given with error. U.S.S.R. Comput. Math. Math. Phys., 1977, vol. 17, no. 5, pp. 1161-1171.

3. Sendov B. X. A modified Steklov function. C. R. Acad. Bulg. Sci., 1983, vol. 134, no. 2, pp. 355379.

4. Khromov A. P., Khromova G. V. On a modification of the Steklov operator. Modern Problems in Function Theory and Applications: Abstracts of Papers of Saratov Winter School, Saratov, Saratov Univ. Press, 2010, pp. 181 (in Russian).

5. Khromov A. P., Khromova G. V. A family of operators with discontinuous ranges and approximation and restoration of continuous functions. Comput. Math. Math. Phys., 2013, vol. 53, no. 10, pp. 16031609. DOI: 10.1134/S0965542513100096.

6. Khromov A. P., Khromova G. V. Discontinuous

Steklov operators in the problem of uniform approximation of derivatives on an interval. Comput. Math. Math. Phys., 2014, vol. 54, no. 9, pp. 57-62. DOI: 10.1134/S0965542514090085.

7. Хромов A. A., Khromova G. V. The solution of the problem of determining the density of heat sources in a rod, Izv. Saratov Univ. (N.S.), Ser. Math. Mech. Inform., 2015, vol. 15, no. 3, pp. 309-314. DOI: 10.18500/1816-9791-2015-15-3-309-314.

8. Khromova G. V. Regularization of Abel Equation with the Use of Discontinuous Steklov Operator, Izv. Saratov Univ. (N.S.), Ser. Math. Mech. Inform., 2014, vol. 14, no. 4, pp. 597-601.

9. Khromova G. V. On uniform approximations to the solution of the Abel integral equation. Comput. Math. Math. Phys., 2015, vol. 55, no. 10, pp. 17031712. DOI: 10.1134/S0965542515100139.

10. Nathanson I. P. Theory of functions of a real variable. St. Petersburg, Lan', 2013, 560 p. (in Russian).

Please cite this article in press as:

Khromova G. V. On Operators with Discontinuous Range. Izv. Saratov Univ. (N. S.), Ser. Math. Mech. Inform., 2016, vol. 16, iss. 3, pp. 298-302 (in Russian). DOI: 10.18500/1816-9791-2016-16-3-298-302.

\section{УДК 591.65}

\section{О ПРЕДЕЛЬНОМ ЗНАЧЕНИИ ОСТАТОЧНОГО ЧЛЕНА КОНСТАНТЫ ЛЕБЕГА, СООТВЕТСТВУЮЩЕЙ ТРИГОНОМЕТРИЧЕСКОМУ ПОЛИНОМУ ЛАГРАНЖА}

\section{И. А. Шакиров}

Шакиров Искандер Асгатович, кандидат фризико-математических наук, проректор по дополнительному образованию, Набережночелнинский государственный педагогический университет, iskander@tatngpi.ru

Изучается поведение константы Лебега тригонометрического полинома Лагранжа, интерполирующего периодическую фрункцию в нечетном числе узлов. Найдено предельное значение остаточного члена, входящего в известную асимптотическую фрормулу для этой константы. Специальное представление остаточного члена позволило установить его строгое убывание. На этой основе для константы Лебега получена неулучшаемая равномерная двусторонняя оценка логарисрмическими фрункциями. Решены экстремальные задачи, связанные с наилучшим приближением константы Лебега: указаны вполне определенные элементы наилучшего приближения и значение наилучшего приближения.

Ключевые слова: полином Лагранжа, квадратурная фрормула, константа Лебега, экстремальная задача, аппроксимация.

DOI: 10.18500/1816-9791-2016-16-3-302-310 


\section{ВВЕДЕНИЕ}

Приближение периодической функции тригонометрическими полиномами Лагранжа на практике осуществляется по равноотстоящим узлам, что дает выгодное с вычислительной точки зрения и близкое к оптимальному приближение исходной функции. При этом принципиальное значение имеют соответствующие им фундаментальные характеристики (функции и константы Лебега). Они впервые были введены X. Лебегом в начале XX века в процессе изучения допущенной погрешности при таком способе приближения функции. Существенный вклад в развитие данного направления внесли Г. Фабер, Л. Фейер, С. Н. Бернштейн, Ю. Марцинкевич, Н. П. Натансон, С. М. Никольский, А. Зигмунд, В. Л. Гончаров [1], Н. И. Ахиезер, А. Ф. Тиман, А. Х. Турецкий [2], А. А. Привалов [3], Т. Ривлин, К. И. Бабенко и многие другие математики. В прошлом веке активные исследования в этой области проводили советские математики. В частности, их научные интересы были связаны с поиском более точных нижних и верхних оценок для фундаментальных характеристик, изучением их асимптотических поведений. В рамках данной работы рассмотрены и исследованы некоторые случаи тригонометрического интерполирования по равномерно распределенным на периоде узлам.

Известно, что тригонометрическому полиному Лагранжа

$$
L_{n}(x, t)=\frac{2}{2 n+1} \sum_{k=1}^{2 n+1} x\left(\tilde{t}_{k}\right) D_{n}\left(\tilde{t}_{k}-t\right) \quad\left(D_{n}(t)=\frac{\sin (n+0.5) t}{2 \sin 0.5 t}, \quad \tilde{t}_{k}=\frac{2 \pi}{2 n+1} k, \quad k=\overline{1, N}\right),
$$

определенному в нечетном числе $N=2 n+1(n \in \mathbb{N})$ узлов интерполяции $\tilde{t}_{k}$, соответствует константа Лебега [4, с. 46]:

$$
\begin{gathered}
\lambda_{n}=\frac{1}{2 n+1}\left(1+2 \sum_{k=1}^{n} \operatorname{cosec} \frac{2 k-1}{4 n+2} \pi\right)= \\
=\frac{1}{2 n+1}\left(1+2 \sum_{k=1}^{n} \operatorname{cosec}\left(t_{k}-\frac{\pi}{2(2 n+1)}\right)\right) \quad\left(t_{k}=\frac{\pi k}{2 n+1}\right),
\end{gathered}
$$

где $n$ - степень полинома (1). При $n=0$ имеем тривиальный случай приближения исходной функции $\left(x(t) \approx L_{0}(x, t) \equiv x(2 \pi), \lambda_{0}=1\right)$, который далее исключим из рассмотрения.

Для константы (2) имеет место известное асимптотическое равенство:

$$
\lambda_{n}=(2 / \pi) \ln n+O(1), \quad n \rightarrow \infty \quad\left(O_{n} \equiv O(n)=\lambda_{n}-(2 / \pi) \ln n, \quad n \in \mathbb{N}\right),
$$

где $O_{n}$ - остаточный член константы Лебега, соответствующий фиксированному значению параметра $n, \beta_{0}=\lim _{n \rightarrow \infty} O_{n}$ - его предельное значение, и они являются неопределенными числами из некоторого ограниченного интервала.

Проблемы верхней и нижней оценки константы (2), ее аналогов в случае интерполирования по четному числу узлов и по узлам Чебышева, многие другие вопросы подробно изучены в монографиях $[1-3,5]$, непосредственно посвященных теории интерполирования. Среди работ, связанных с более детальным исследованием соответствующих им остаточных членов (нахождением малых интервалов, к которым они принадлежат) и посвященных изучению их предельных значений, отметим статьи [6-10]. Заметим, что до сих пор не все составляющие константы Лебега (3) исследованы в полной мере; их более детальному рассмотрению и посвящена данная работа.

Получены следующие результаты:

- приведены специальные представления для остаточного члена, позволяющие установить строгое убывание последовательности $\left(O_{n}\right)$ и строгое возрастание $\left(\lambda_{n}\right)$;

- найдено предельное значение последовательности $\left(O_{n}\right)$ :

$$
\beta_{0}=\lim _{n \rightarrow \infty} O_{n}=(2 / \pi)[\gamma+\ln (16 / \pi)]=1.403794027 \ldots \quad(\gamma-\text { константа Эйлера }) ;
$$

- решена экстремальная задача вида

$$
\sup _{c \in \mathbb{R}}\left\{c \mid \lambda_{n} \geqslant c+(2 / \pi) \ln n, n \in \mathbb{N}\right\}=\frac{2}{\pi}\left(\ln \frac{16}{\pi}+\gamma\right)=\beta_{0} ;
$$


- с учетом результатов предыдущих пунктов для константы Лебега получена неулучшаемая равномерная двусторонняя оценка:

$$
(2 / \pi)[\gamma+\ln (16 / \pi)]+(2 / \pi) \ln n \leqslant \lambda_{n} \leqslant 5 / 3+(2 / \pi) \ln n \quad(n \in \overline{\mathbb{N}}=\mathbb{N} \cup\{+\infty\})
$$

- решены экстремальные задачи, связанные с поиском элемента наилучшего приближения для константы $\lambda_{n}$ и соответствующего наилучшего приближения.

Отметим, что часть результатов данной работы опубликована в работе [11] автора.

\section{1. ВСПОМОГАТЕЛЬНЫЕ ОПРЕДЕЛЕНИЯ И ЛЕММЫ}

В ходе доказательства основных теорем существенно используются следующие определение [10] и леммы.

Определение. Строго монотонную функцию $\varphi=\varphi(n)(n \in \tilde{D} \subseteq \mathbb{N})$ дискретного аргумента, имеющую малое изменение $\delta$ области значений $R(\varphi)$, назовем функцицй, имеющей малую монотонную вариацию, и класс таких функций обозначим через $V_{\delta}^{ \pm}$, где знак плюс используется в случае возрастания функции в области $\tilde{D}$, а минус - при ее убывании; $\delta=\sup \{\varphi(n) \mid n \in \tilde{D}\}-\inf \{\varphi(n) \mid n \in \tilde{D}\}$.

Введем в рассмотрение функцию

$$
y=y(t)=1 / \sin t-1 / t \quad\left(t \in D=[0, \pi / 2], \quad y(0)=y(0+)=\lim _{t \rightarrow 0+} y(t)=0\right)
$$

и составим соответствующую ей интегральную сумму вида

$$
\begin{gathered}
S_{n}=S(n)=\sum_{k=1}^{n}\left(\frac{1}{\sin \left(t_{k}-\pi /(4 n+2)\right)}-\frac{1}{t_{k}-\pi /(4 n+2)}\right) \frac{\pi}{2 n+1} \\
\left(t_{k}=\frac{\pi k}{2 n+1} \in[0, \pi / 2], \quad n \in \mathbb{N}\right) .
\end{gathered}
$$

Лемма 1. Функииональная зависимость (5) является неотрицательной, строго возрастающей и выпуклой вниз функцией в области D. Соответствующая сумме (6) последовательность $\left(S_{n}\right)$ строго возрастает и стремится к своему предельному значению $\ln (4 / \pi)$, т.е.

$$
S_{n}<S_{n+1} \quad \forall n \in \mathbb{N}, \quad \lim _{n \rightarrow \infty} S_{n}=\ln (4 / \pi) .
$$

Доказательство. Для первой и второй производных функции (5) выполняются неравенства

$$
\begin{gathered}
y^{\prime}=\frac{1}{t^{2} \sin ^{2} t}\left(\frac{1}{6} t^{4}+\frac{1}{360} t^{6}+\ldots\right)=\left(\frac{t}{\sin t}\right)^{2}\left(\frac{1}{6}+\frac{1}{360} t^{2}+\ldots\right)>0 \quad(t \in(0, \pi / 2]), \\
y^{\prime \prime}=\frac{1}{t^{3} \sin ^{3} t}\left(\frac{7}{60} t^{7}-\frac{47}{2520} t^{9}+\ldots\right)=t\left(\frac{t}{\sin t}\right)^{3}\left(\frac{7}{60}-\frac{47}{2520} t^{2}+\ldots\right)>0 \quad(t \in(0, \pi / 2]),
\end{gathered}
$$

причем $y^{\prime}(0)=y^{\prime}(0+)=1 / 6, y^{\prime \prime}(0)=y^{\prime \prime}(0+)=0, R(y)=[0,1-2 / \pi]$. Эти сведения достаточны для завершения доказательства первой части леммы.

Относительно системы узлов $t_{k}=\pi k /(2 n+1)(k=\overline{0, n})$ сумма (6) представляет собой квадратурную формулу средних прямоугольников для определенного интеграла $I_{n}=I(n)=\int_{0}^{t_{n}}(1 / \sin t-1 / t) d t$ с верхней переменной границей $t_{n}$, для которой верны соотношения

$$
t_{n}<t_{n+1}<\frac{\pi}{2} \quad \forall n \in \mathbb{N} \quad\left(t_{n}=\frac{\pi}{2}-\frac{\pi}{2(2 n+1)}\right), \quad \lim _{n \rightarrow \infty} t_{n}=\pi / 2 .
$$

Расширение области интегрирования при неограниченном увеличении параметра $n$ от промежутка $\left[0, t_{n}\right]$ до $[0, \pi / 2]$ и установленные выше свойства подынтегральной функции обеспечивают выполнение соотношений

$$
I_{n}<I_{n+1}<I \quad \forall n \in \mathbb{N}, \quad I=\lim _{n \rightarrow \infty} I_{n}=\int_{0}^{\pi / 2}(1 / \sin t-1 / t) d t=\ln (4 / \pi) .
$$


Согласно лемме 1 из [6] для класса выпуклых вниз в области своего определения функций средние суммы Римана монотонно возрастают независимо от поведения их третьих производных. В нашем случае неотрицательная функция (5), у которой первая и вторая производные также неотрицательны, удовлетворяет условиям указанной леммы. Поэтому для соответствующей квадратурной формулы средних прямоугольников (6) с учетом вышеизложенного выполняются неравенства $S_{n}<S_{n+1}, n \in \mathbb{N}$, а также дополнительные соотношения:

$$
S_{n}<I_{n} \quad(n \in \mathbb{N}), \lim _{n \rightarrow \infty} S_{n}=S=\int_{0}^{\pi / 2}(1 / \sin t-1 / t) d t=\ln (4 / \pi)=0.241564475 \ldots,
$$

где $S_{1}=2 \pi / 3-2=0.094395102 \ldots, \quad S_{2}=\frac{\pi}{5}\left(\operatorname{cosec} \frac{\pi}{10}+\operatorname{cosec} \frac{3 \pi}{10}\right)-\frac{8}{3}=0.143259225 \ldots$, $S_{3}=\frac{\pi}{7}\left(\operatorname{cosec} \frac{\pi}{14}+\operatorname{cosec} \frac{3 \pi}{14}+\operatorname{cosec} \frac{5 \pi}{14}\right)-\frac{46}{15}=0.168164392 \ldots$ и т.д.

Справедливость леммы 1 установлена полностью.

Лемма 2. Разность $\sum_{k=1}^{\infty} 1 /(k-0.5)-\sum_{k=1}^{\infty} 1 / k$ двух расходящихся числовых рядов $\sum_{k=1}^{\infty} 1 /(k-0.5)$ и $\sum_{k=1}^{\infty} 1 / k$ является сходящимся рядом, для суммы s которого верно следующее равенство:

$$
s=\lim _{n \rightarrow \infty} s_{n}=\ln 4 \quad\left(s_{n}=\sum_{k=1}^{n} 1 /(k-0.5)-\sum_{k=1}^{n} 1 / k=\sum_{k=1}^{n} 1 /[k(2 k-1)], \quad n \in \mathbb{N}\right)
$$

где $\left(s_{n}\right)$ - строго возрастающая числовая последовательность.

Доказательство. Над известным разложением в степенной ряд функции $y=\ln (1-t)(t \in[0,1))$ проведем следующие преобразования:

$$
\begin{gathered}
\ln (1-t)=-t-\frac{1}{2} t^{2}-\frac{1}{3} t^{3}-\frac{1}{4} t^{4}-\frac{1}{5} t^{5}-\ldots \quad \Leftrightarrow \quad \Leftrightarrow \quad \Leftrightarrow \quad \Leftrightarrow \quad \Rightarrow \quad(t \in(0,1)) \quad \Rightarrow \\
\Leftrightarrow-\frac{1}{t} \ln (1-t)=1+\frac{1}{2} t+\frac{1}{3} t^{2}+\frac{1}{4} t^{3}+\frac{1}{5} t^{4}+\ldots \quad \ln \left(1-t^{2}\right)=1+\frac{1}{2} t^{2}+\frac{1}{3} t^{4}+\frac{1}{4} t^{6}+\frac{1}{5} t^{8}+\ldots \quad t \quad d t . \\
\Rightarrow \quad-\int_{0}^{1} \frac{1}{t^{2}} \ln \left(1-t^{2}\right) d t=\int_{0}^{1}\left(1+\frac{1}{2} t^{2}+\frac{1}{3} t^{4}+\frac{1}{4} t^{6}+\frac{1}{5} t^{8}+\ldots\right) d t
\end{gathered}
$$

Применяя формулу интегрирования по частям к несобственному интегралу в левой части последнего равенства, после некоторых вычислений найдем его значение, равное $\ln 4$. В правой части равенства после интегрирования получим ряд $\sum_{k=1}^{\infty} 1 /[k(2 k-1)]$, последовательность частных сумм которого, очевидно, монотонно возрастает. Лемма доказана.

\section{2. ОСНОВНЫЕ РЕЗУЛЬТАТЫ}

Для большинства встречающихся в приложениях операторов соответствующие им константы Лебега либо равномерно ограничены, либо ведут себя как логарифмическая функция натурального аргумента. В нашем случае поведения последовательностей $\left(\lambda_{n}\right),\left(O_{n}\right)$ и их предельных значений будут полностью определены следующими теоремами.

Теорема 1. Для остаточного илена $O_{n}=\lambda_{n}-(2 / \pi) \ln n$ имеет место представление

$$
O_{n}=O(n)=(2 / \pi)\left[S_{n}+s_{n}+\gamma_{n}+\pi /(4 n+2)\right] \quad(n \in \mathbb{N}),
$$

где последовательности $\left(S_{n}\right),\left(s_{n}\right)$ определень в (6), (8) соответственно, а числа $\gamma_{n}$ определяют известную константу Эйлера:

$$
\gamma=\lim _{n \rightarrow \infty} \gamma_{n}=0.5772156649 \ldots \quad\left(\gamma_{n}=\sum_{k=1}^{n} 1 / k-\ln n\right)
$$


Доказательство. Преобразуем константу Лебега (2), используя при этом соотношения (6), (8)(10):

$$
\begin{gathered}
\lambda_{n}=\frac{1}{2 n+1}+\frac{2}{\pi} \sum_{k=1}^{n} \frac{\pi}{2 n+1} \operatorname{cosec}\left(t_{k}-\frac{\pi}{4 n+2}\right)= \\
=\frac{2}{\pi}\left\{\left[\sum_{k=1}^{n} \frac{1}{\sin \left(t_{k}-\pi /(4 n+2)\right)} \cdot \frac{\pi}{2 n+1}-\sum_{k=1}^{n} \frac{1}{t_{k}-\pi /(4 n+2)} \cdot \frac{\pi}{2 n+1}\right]+\right. \\
\left.+\sum_{k=1}^{n} \frac{1}{t_{k}-\pi /(4 n+2)} \cdot \frac{\pi}{2 n+1}+\frac{\pi}{2(2 n+1)}\right\}=\frac{2}{\pi}\left[S_{n}+\sum_{k=1}^{n} \frac{1}{k-1 / 2}+\frac{\pi}{4 n+2}\right]= \\
=\frac{2}{\pi}\left[S_{n}+\left(\sum_{k=1}^{n} \frac{2}{2 k-1}-\sum_{k=1}^{n} \frac{1}{k}\right)+\sum_{k=1}^{n} \frac{1}{k}+\frac{\pi}{4 n+2}\right]= \\
=\frac{2}{\pi}\left[S_{n}+\sum_{k=1}^{n} \frac{1}{k(2 k-1)}+\gamma_{n}+\ln n+\frac{\pi}{4 n+2}\right]= \\
=\frac{2}{\pi} \ln n+\frac{2}{\pi}\left(S_{n}+s_{n}+\gamma_{n}+\frac{\pi}{4 n+2}\right)=(2 / \pi) \ln n+O_{n} \quad\left(t_{k}=\pi k /(2 n+1)\right) .
\end{gathered}
$$

Теорема доказана.

Далее, используя теорему 1, приведем простейшее доказательство строгого возрастания последовательности констант Лебега (2) (известный факт [12, с. 125]). Затем установим строгое убывание последовательности (9), и найдем ее предельное значение.

Теорема 2. Последовательность $\left(O_{n}\right)$ строго убывает $\left(O_{n} \in V_{\delta}^{-}\right)$, а последовательность $\left(\lambda_{n}\right)$ строго возрастает.

Доказательство. Вначале докажем вторую часть теоремы. Для этого проверим выполнение неравенства $\lambda_{n+1}>\lambda_{n}$ для произвольного $n \in \mathbb{N}$, используя при этом одно из представлений для константы Лебега, полученное в ходе доказательства предыдущей теоремы, и неравенство (7):

$$
\begin{gathered}
\lambda_{n+1}-\lambda_{n}=\frac{2}{\pi}\left[\left(S_{n+1}+\sum_{k=1}^{n+1} \frac{1}{k-1 / 2}+\frac{\pi}{4(n+1)+2}\right)-\left(S_{n}+\sum_{k=1}^{n} \frac{1}{k-1 / 2}+\frac{\pi}{4 n+2}\right)\right]= \\
=\frac{2}{\pi}\left[\left(S_{n+1}-S_{n}\right)+\left(\sum_{k=1}^{n+1} \frac{2}{2 k-1}-\sum_{k=1}^{n} \frac{2}{2 k-1}\right)+\frac{\pi}{2}\left(\frac{1}{2 n+3}-\frac{1}{2 n+1}\right)\right]= \\
=\frac{2}{\pi}\left[\left(S_{n+1}-S_{n}\right)+\frac{4 n+6-\pi}{(2 n+1)(2 n+3)}\right]>0 \quad(n \in \mathbb{N}),
\end{gathered}
$$

где обе части слагаемого в квадратных скобках положительны.

Для доказательства первой части теоремы используем полученное в работе [10, формула (18)] двойное неравенство

$$
(11 / 6) \alpha_{n}+\varphi_{n}+(2 / \pi) \ln n<\lambda_{n}<2 \alpha_{n}+\varphi_{n}+(2 / \pi) \ln n(n \in \mathbb{N}),
$$

которое представим в виде

$$
\begin{aligned}
& (11 / 6) \alpha_{n}+\varphi_{n}<\lambda_{n}-(2 / \pi) \ln n<2 \alpha_{n}+\varphi_{n} \quad \Leftrightarrow \\
& \Leftrightarrow \quad(11 / 6) \alpha_{n}+\varphi_{n}<O_{n}<2 \alpha_{n}+\varphi_{n} \quad(n \in \mathbb{N}),
\end{aligned}
$$

где $\alpha_{n} \equiv 1 /[(2 n+1) \sin (\pi /(4 n+2))], \varphi_{n} \equiv \frac{2}{\pi} \ln \left[\left(2+\frac{1}{n}\right) \alpha_{n} \cos \left(\frac{\pi}{4 n+2}\right)\right] \quad(n \in \mathbb{N})$.

Согласно леммам 1 и 2 из [10] для функций натурального аргумента $\alpha_{n}=\alpha(n), \varphi_{n}=\varphi(n)$ верны соотношения $\alpha_{n} \in V_{\delta}^{-}, \varphi_{n} \in V_{\delta}^{-}$. Ясно, что их линейные комбинации $\psi_{n} \equiv(11 / 6) \alpha_{n}+\varphi_{n}, \bar{\psi}_{n} \equiv 2 \alpha_{n}+\varphi_{n}$ также являются строго убывающими функциями $\left(\psi_{n}, \bar{\psi}_{n} \in V_{\delta}^{-}\right)$; для их образов и вариаций верны соотношения

$$
R\left(\psi_{n}\right)=\left(\frac{11}{3 \pi}+\frac{2}{\pi} \ln \frac{4}{\pi}, \frac{11}{9}+\frac{1}{\pi} \ln 3\right] \subset(1.320920,1.571922), \quad \delta\left(\psi_{n}\right)=0.251000 \ldots,
$$




$$
R\left(\bar{\psi}_{n}\right)=\left(\frac{4}{\pi}+\frac{2}{\pi} \ln \frac{4}{\pi}, \frac{4}{3}+\frac{1}{\pi} \ln 3\right] \subset(1.427024,1.683033), \quad \delta\left(\bar{\psi}_{n}\right)=0.256008 \ldots
$$

Эти сведения, неравенство (11) и теорема о трех последовательностях позволяют утверждать, что остаточный член $O_{n} \in V_{\delta}^{-}\left(R\left(O_{n}\right)=\left(\beta_{0}, 5 / 3\right], \delta\left(O_{n}\right)=0.262872 \ldots\right)$. Следовательно, строго возрастающая функция $\lambda_{n}=\lambda(n) \quad(n \in \mathbb{N})$ состоит из логарифмической части $(2 / \pi) \ln n$ и строго убывающего остаточного члена $O_{n}\left(O_{n} \in V_{\delta}^{-}\right)$вида (9).

Теорема 2 полностью доказана.

Теперь, используя представление (9) для остаточного члена $O_{n}$, найдем его предельное значение $\beta_{0}$.

Теорема 3. Для последовательности $\left(O_{n}\right)$ имеет место следующее предельное равенство:

$$
\beta_{0}=\lim _{n \rightarrow \infty} O_{n}=\frac{2}{\pi}\left(\gamma+\ln \frac{16}{\pi}\right)=1.403794027 \ldots .
$$

Доказательство. Переходя в (9) к пределу и используя при этом соотношения (7), (8), (10), легко получим справедливость (12):

$$
\begin{aligned}
\lim _{n \rightarrow \infty} O_{n} & =\frac{2}{\pi}\left[\lim _{n \rightarrow \infty} S_{n}+\lim _{n \rightarrow \infty} s_{n}+\lim _{n \rightarrow \infty} \gamma_{n}+\lim _{n \rightarrow \infty} \pi /(4 n+2)\right]= \\
& =\frac{2}{\pi}\left(\ln \frac{4}{\pi}+\ln 4+\gamma\right)=\frac{2}{\pi}\left(\gamma+\ln \frac{16}{\pi}\right)=\beta_{0},
\end{aligned}
$$

причем

$$
R\left(O_{n}\right)=\left(\frac{2}{\pi} \gamma+\frac{2}{\pi} \ln \frac{16}{\pi}, \frac{5}{3}\right] \subset(1.403794,1.666667), \quad \delta=\delta\left(O_{n}\right)=0.262872 \ldots .
$$

Следствие 1. Для константы Лебега (2) имеет место представление

$$
\lambda_{n}=\beta_{0}+(2 / \pi) \ln n+\bar{O}_{n} \quad\left(\bar{O}_{n}=O_{n}-\beta_{0}, \quad n \in \mathbb{N}\right),
$$

где для нового остаточного илена $\bar{O}_{n}$ выполнены соотношения

$$
\bar{O}_{n} \in V_{\delta}^{-}, \quad R\left(\bar{O}_{n}\right)=\left(0,5 / 3-\beta_{0}\right] \subset(0,0.262873), \quad \delta\left(\bar{O}_{n}\right)=0.262872 \ldots .
$$

Доказательство. Найденное ранее предельное значение $\beta_{0}$ позволяет выразить константу Лебега через логарифмическую функцию $\beta_{0}+(2 / \pi) \ln n$ (главную часть) и новый остаточный член $\bar{O}_{n}$, т. е. представить ее в виде (14). Так как $O_{n} \in V_{\delta}^{-}$(см. теорему 2), то линейно выраженный через него остаточный член $\bar{O}_{n}$ также принадлежит этому же классу. Теперь из (13), (14) легко следует справедливость соотношений (15).

Следствие 2. Константа $\beta_{0}$ является единственным решением әкстремальной задачи

$$
\sup \left\{c \in \mathbb{R} \mid \lambda_{n} \geqslant c+(2 / \pi) \ln n, n \in \mathbb{N}\right\}=\beta_{0}=1.403794027 \ldots,
$$

причем в нижней оценке $\beta_{0}+(2 / \pi) \ln n \leqslant \lambda_{n} \quad(n \in \overline{\mathbb{N}})$ для константы Лебега равенство достигается лишь при $n=+\infty \in \overline{\mathbb{N}}, m . e$.

$$
\lim _{n \rightarrow \infty} \lambda_{n}=\beta_{0}+\lim _{n \rightarrow \infty}[(2 / \pi) \ln n] .
$$

Доказательство. Выполнение утверждений следствия 2 (см. также (4)) обеспечивает свойство строгой монотонности последовательности $O_{n} \quad\left(O_{n} \in V_{\delta}^{-}\right)$.

Теорема 4. Константу Лебега $\lambda_{n}=\lambda(n)$ наилучшим образом приближает логарифмическая функиия:

$$
\mu_{n}^{*}=\mu^{*}(n)=\frac{2}{\pi} \ln n+\frac{5}{6}+\frac{\gamma}{\pi}+\frac{1}{\pi} \ln \frac{16}{\pi} \quad(n \in \mathbb{N}),
$$

т.е. она является решением следующей экстремальной задачи:

$$
\inf _{b \in[0, \infty)} \sup _{n \in \mathbb{N}}\left|\lambda_{n}-b-\frac{2}{\pi} \ln n\right|=\sup _{n \in \mathbb{N}}\left|\lambda_{n}-\mu_{n}^{*}\right|=\frac{5}{6}-\frac{\gamma}{\pi}-\frac{1}{\pi} \ln \frac{16}{\pi}=0.131436319 \ldots=\varepsilon^{*},
$$

где $\varepsilon^{*}$ - наилучшее равномерное (дискретное) приближение $\lambda(n)$ логарифмическими функциями $[13$, с. 9]. 
Доказательство. Полученные в теореме 3 и в двух ее следствиях результаты обеспечивают выполнение следующего двойного неравенства для константы (2):

$$
(2 / \pi)[\gamma+\ln (16 / \pi)]+(2 / \pi) \ln n \leqslant \lambda_{n} \leqslant 5 / 3+(2 / \pi) \ln n \quad(n \in \overline{\mathbb{N}}),
$$

где нижнее равенство достигается только при предельном значении параметра $n(n=+\infty)$, а верхнее - при $n=1$.

Согласно (17) все значения дискретной функции $z=\lambda(n) \quad(n \in \overline{\mathbb{N}})$, рассматриваемой в декартовой системе координат $n O z$, находятся строго между двумя вполне определенными логарифмическими функциями (совпадение значений $\lambda(n)$ и указанных функций имеет место лишь на концах их области определения, т.е. при $n=1$ и $n=+\infty)$. Ясно, что их полусумма $\mu_{n}^{*}=\mu^{*}(n)=\frac{2}{\pi} \ln n+\frac{5}{6}+\frac{\gamma}{\pi}+\frac{1}{\pi} \ln \frac{16}{\pi}=\frac{2}{\pi} \ln n+1.535230346 \ldots$ является элементом наилучшего равномерного приближения для $\lambda_{n}$ среди всевозможных логарифмических функций вида $b+a \ln n$ $(a, b \in[0, \infty), n \in \mathbb{N})$.

Проверка справедливости соотношения (16) требует лишь элементарных технических расчетов.

Замечание. Рассматривая экстремальную задачу (16) на различных вложенных друг в друга подмножествах множества натуральных чисел $\mathbb{N}$ и используя при этом результаты теоремы 2 относительно последовательностей $\left(O_{n}\right),\left(\lambda_{n}\right)$, можно сколько угодно уменьшать значение погрешности $\varepsilon^{*}$, возникающей в процессе приближенного вычисления константы Лебега согласно формуле $\lambda_{n} \approx \mu_{n}^{*}$ $(n \in \mathbb{N})$.

Например, для константы (2) при $\mathbb{N}_{0}=\{4,5,6,7, \ldots\} \subset \mathbb{N}$ имеем:

$$
\begin{gathered}
\lambda_{1}=\frac{5}{3}=1.666666666 \ldots \\
\lambda_{2}=\frac{1}{5}+\frac{2}{5} \operatorname{cosec} \frac{\pi}{10}+\frac{2}{5} \operatorname{cosec} \frac{3 \pi}{10}=1.988854381 \ldots, \\
\lambda_{3}=\frac{1}{7}+\frac{2}{7} \operatorname{cosec} \frac{\pi}{14}+\frac{2}{7} \operatorname{cosec} \frac{3 \pi}{14}+\frac{2}{7} \operatorname{cosec} \frac{5 \pi}{14}=2.202214555 \ldots
\end{gathered}
$$

(они вычислены точно). Остальные значения константы Лебега вычислим приближенно $\left(\lambda_{n} \approx \bar{\mu}_{n}\right.$, $n \geqslant 4)$, используя при этом логарифмическую функцию:

$$
\bar{\mu}_{n}=\bar{\mu}(n)=\frac{2}{\pi} \ln n+\frac{1}{2}\left(-\frac{2}{\pi} \ln 3+\beta_{0}+2.202214555 \ldots\right)=\frac{2}{\pi} \ln n+1.453305138 \ldots \quad(n \geqslant 4)
$$

наилучшего равномерного приближения для $\lambda_{n}=\lambda(n) \quad\left(n \in \mathbb{N}_{0}\right)$. Она построена согласно алгоритму, использованному в ходе доказательства теоремы 4. В этом случае для модифицированной экстремальной задачи имеем:

$$
\begin{gathered}
\inf _{b \in[0, \infty)} \sup _{n \in \mathbb{N}_{0}}\left|\lambda_{n}-\frac{2}{\pi} \ln n-b\right|=\sup _{n \in \mathbb{N}_{0}}\left|\lambda_{n}-\bar{\mu}_{n}\right|=\frac{1}{2}\left(-\frac{2}{\pi} \ln 3-\beta_{0}+2.202214555 \ldots\right)= \\
=0.049511111 \ldots=\bar{\varepsilon} .
\end{gathered}
$$

Таким образом, выбором подмножества $\mathbb{N}_{0} \subset \mathbb{N}$ и соответствующей функции $\bar{\mu}(n)\left(n \in \mathbb{N}_{0}\right)$ ранее найденная погрешность $\varepsilon^{*}$ уменьшена более чем в 2.5 раза $\left(\varepsilon^{*} / \bar{\varepsilon}=2.654683 \ldots\right)$.

\section{Библиографический список}

1. Гончаров В. Л. Теория интерполирования и приближения функций. М. : Гостехиздат, 1954. 328 с.

2. Турец̧кий $A$. X. Теория интерполирования в задачах. Минск : Вышэйшая школа, 1968. 320 с.

3. Привалов A. А. Теория интерполирования функций : в 2 кн. Саратов : Изд-во Саратов. ун-та, 1990. Кн. 1. 230 с.; Кн. 2. С. 231-424.

4. Дзядык В. К. Аппроксимационные методы решения дифференциальных и интегральных уравнений. Киев : Наук. думка, 1988. 304 с.
5. Szabados J., Vertesi P. Interpolation of Functions. Singapore: World Scientific, 1990. 305 p.

6. Rivlin T. J. The Lebesgue constants for polynomial interpolation // Functional Analysis and its Application, Int. Conf., Madras, 1973 (eds. H. G. Gamier et al.). Berlin : Springer-Verlag, 1974. P. 422-437.

7. Brutman L. Lebesgue functions for polynomial interpolation - a survey // The heritage of P. L. Chebyshev : a Festschrift in honor of the 70th birthday 
of T. J. Rivlin. Ann. Numer. Math. 1997. Vol. 4, № 1-4. P. 111-127.

8. Vértesi $P$. On the Lebesgue function and Lebesgue constant : a tribute to Paul Erdös // Paul Erdös and his mathematics, I (Budapest, 1999), Bolyai Soc. Math. Stud. Vol. 11. Budapest : János Bolyai Math. Soc., 2002. P. 705-728.

9. Simon J. S. Lebesgue constants in polynomial interpolation //Annal. Math. et Inf. 2006. Vol. 33. P. 109-123.

10. Шакиров И. А. О влиянии выбора узлов лагранжевой интерполяции на точные и приближенные значения констант Лебега // Сиб. матем. журн. 2014. Т. 55, № 6. С. 1404-1423.

11. Шакиров И. А. О значении неопределенной величины в асимптотической формуле для константы Лебега // Современные проблемы теории функций и их приложения : материалы 18-й междунар. Сарат. зимней школы. Саратов : ООО Изд-во «Научная книга», 2016. С. 322-326.

12. Зигмунд А. Тригонометрические ряды : в 2 т. Т. 1. М. : Мир, 1965. 616 с.

13. Корнейчук Н. П. Экстремальные задачи теории приближения. М. : Наука, 1976. 320 с.

\title{
Образец для цитирования:
}

Шакиров И. А. О предельном значении остаточного члена константы Лебега, соответствующей тригонометрическому полиному Лагранжа // Изв. Сарат. ун-та. Нов. сер. Сер. Математика. Механика. Информатика. 2016. Т. 16, вып. 3. С. 302-310. DOI: 10.18500/1816-9791-2016-16-3-302-310.

\section{On a Limit Value of a Remainder of the Lagrange Constant Corresponding to the Lagrange Trigonometrical Polynomial}

\begin{abstract}
I. A. Shakirov
Iskander A. Shakirov, Naberezhnye Chelny State Pedagogical University, 28, Nizametdinov st., 423806, Naberezhniye Chelny, Tatarstan, Russia, iskander@ tatngpi.ru

The behavior of Lebesgue constant of a trigonometrical Lagrange polynomial interpolating the periodic function in an odd number of clusters is studied. The limit value of the remainder in the known asymptotic formula for this constant is found. A special representation of a remainder allowed us to establish its strict decreasing. On this basis, for a Lebesgue constant, a non-improvable uniform bilateral logarithmic function estimate is received. The extremum problems related to the best approximation of a constant of Lebesgue are solved: quite particular elements of the best approximation and the value of the best approximation are specified.
\end{abstract}

Key words: Lagrange polynomial, quadrature formula, Lebesgue constant, extremum problem, approximation.

\section{References}

1. Goncharov V. L. Interpolation theory and approximations of functions. Moscow, Gostekhizdat, 1954, 328 p. (in Russian)

2. Turetskii A. Kh. Teoriia interpolirovaniia v zadachakh [Theory of interpolation in problems]. Minsk, Vysheishaia shkola, 1968, 320 p. (in Russian).

3. Privalov A. A. Teoriya interpolirovaniya funktsi?. Kniga 1, 2 [Theory of interpolation of functions. Book 1, 2]. Saratov, Saratov Univ. Press, 1990. Book 1 : 230 p.; Book 2 : pp. 231-424 (in Russian).

4. Dzyadyk V. K. Approksimatsionnye metody resheniya differentsial?nykh i integral?nykh uravneni? [Approximation methods for solving differential and integral equations]. Kiev, Naukova Dumka, 1988, 304 p. (in Russian).

5. Szabados J., Vertesi P. Interpolation of Functions. Singapore, World Scientific, 1990, 305 p.

6. Rivlin T. J. The Lebesgue constants for polynomial interpolation. Functional Analysis and its Application, Int. Conf., Madras, 1973 (eds. H. G. Gamier et al.), Berlin, Springer-Verlag, 1974, pp. 422-437.

7. Brutman L. Lebesgue functions for polynomial in- terpolation - a survey. The heritage of P. L. Chebyshev : a Festschrift in honor of the 70th birthday of T. J. Rivlin. Ann. Numer. Math., 1997, vol. 4, no. 1-4, pp. 111-127.

8. Vértesi P. On the Lebesgue function and Lebesgue constant : a tribute to Paul Erdös. Paul Erdös and his mathematics, I (Budapest, 1999), Bolyai Soc. Math. Stud., vol. 11, Budapest, János Bolyai Math. Soc., 2002, pp. 705-728.

9. Simon J. S. Lebesgue constants in polynomial interpolation. Annal. Math. et Inf., 2006, vol. 33, pp. 109-123.

10. Shakirov I. A. Influence of the choice of Lagrange interpolation nodes on the exact and approximate values of the Lebesgue constants. Siberian Math. J., 2014, vol. 55, iss. 6, pp. 1144-1160. DOI: 10.1134/S0037446614060184.

11. Shakirov I. A. O znachenii neopredelennoi velichiny $\mathrm{v}$ asimptoticheskoi formule dlia konstanty Lebega [About the value of uncertain quantities in the asymptotic formula for the Lebesgue constants]. Souremennye problemy teorii funktsii $i$ ikh prilozheniia : materialy 18-i mezhdunar. Sarat. zimnei shkoly [Modern problems of function theo- 
ry and their applications : Proc. 18th Intern. Sarat. Winter School]. Saratov, OOO Izd-vo "Nauchnaia kniga", 2016, pp. 322-326.

12. Zygmund A. Trigonometric series: Vol. I, II. Second edition, reprinted with corrections and some additions. London ; New York, Cambridge Univ. Press, 1968, vol. I : 383 p.; vol. II : 364 p. (two volumes bound as one) (Russ. ed. : Zygmund A. Trigonometricheskie riady. Tom 1. Moscow, Mir, 1965, 616 p.)

13. Korneichuk N. P. Ekstremal'nye zadachi teorii priblizheniya [Extremal problems of approximation theory]. Moscow, Nauka, 1976, 320 p. (in Russian).

Please cite this article in press as:

Shakirov I. A. On a Limit Value of a Remainder of the Lagrange Constant Corresponding to the Lagrange Trigonometrical Polynomial. Izv. Saratov Univ. (N. S.), Ser. Math. Mech. Inform., 2016, vol. 16, iss. 3, pp. $302-310$ (in Russian). DOI: 10.18500/1816-9791-2016-16-3-302-310.

УДК 517.587

\title{
ПОЛИНОМЫ, ОРТОГОНАЛЬНЫЕ ПО СОБОЛЕВУ, ПОРОЖДЕННЫЕ МНОГОЧЛЕНАМИ МЕЙКСНЕРА
}

\author{
И. И. Шарапудинов ${ }^{1}$, З. Д. Гаджиева ${ }^{2}$
}

${ }^{1}$ Шарапудинов Идрис Идрисович, заведующий отделом математики и инсрорматики, Дагестанский научный центр РАН; Владикавказский научный центр РАН; Дагестанский государственный педагогический университет, Махачкала, sharapud@mail.ru

${ }^{2}$ Гаджиева Зульсрия Джамалдиновна, научный сотрудник отдела математики и инсрорматики, Дагестанский научный центр РАН, Дагестанский государственный педагогический университет, Махачкала, zulfiya.gadzhieva.1976@ mail.ru

Рассмотрена задача о конструировании полиномов $m_{r, n}^{\alpha}(x, q),(n=0,1, \ldots)$, ортогональных по Соболеву и порожденных классическими полиномами Мейкснера. Эти полиномы могут быть определены с помощью следующих равенств $m_{r, k}^{\alpha}(x, q)=\frac{x^{[k]}}{k !}, x^{[k]}=x(x-1) \cdots(x-k+1), k=0,1, \ldots, r-1$, $m_{r, k+r}^{\alpha}(x, q)=\frac{1}{(r-1) !} \sum_{t=0}^{x-r}(x-1-t)^{[r-1]} m_{k}^{\alpha}(t, q)$, где через $m_{k}^{\alpha}(t, q)$ обозначены полиномы Мейкснера, ортонормированные на сетке $\Omega=\{0,1, \ldots\}$ с весом $\rho(x)=q^{x} \frac{\Gamma(x+\alpha+1)}{\Gamma(x+1)}(1-q)^{\alpha+1}$. Полиномы $m_{r, n}^{\alpha}(x, q)(n=0,1, \ldots)$ образуют ортонормированную систему на $\Omega=\{0,1, \ldots\}$ относительно скалярного произведения типа Соболева следующего вида:

$$
\left\langle m_{r, n}^{\alpha}, m_{r, m}^{\alpha}\right\rangle=\sum_{k=0}^{r-1} \Delta^{k} m_{r, n}^{\alpha}(0, q) \Delta^{k} m_{r, m}^{\alpha}(0, q)+\sum_{j=0}^{\infty} \Delta^{r} m_{r, n}^{\alpha}(j, q) \Delta^{r} m_{r, m}^{\alpha}(j, q) \rho(j) .
$$

Для $m_{r, n}^{\alpha}(x, q)$ мы получили явную фрормулу, содержащую полиномы Мейкснера $M_{n}^{\alpha-r}(x, q)$ :

$$
m_{r, k+r}^{\alpha}(x, q)=\left(\frac{q}{q-1}\right)^{r}\left\{h_{k}^{\alpha}(q)\right\}^{-1 / 2}\left[M_{k+r}^{\alpha-r}(x, q)-\sum_{\nu=0}^{r-1} \frac{A_{r, k, \nu} x^{[\nu]}}{\nu !}\right], \quad k=0,1, \ldots,
$$

где $A_{r, k, \nu}=\left(\frac{q-1}{q}\right)^{\nu} \frac{\Gamma(k+\alpha+1)}{(k+r-\nu) ! \Gamma(\nu-r+\alpha+1)}, M_{n}^{\alpha}(x, q)=\frac{\Gamma(n+\alpha+1)}{n !} \sum_{k=0}^{n} \frac{n^{[k]} x^{[k]}}{\Gamma(k+\alpha+1) k !}\left(1-\frac{1}{q}\right)^{k}$, $h_{n}^{\alpha}(q)=\left(\begin{array}{c}n+\alpha \\ n\end{array}\right) q^{-n} \Gamma(\alpha+1)$.

Ключевые слова: полиномы, ортогональные по Соболеву, полиномы Мейкснера, ортогональные на сетке, приближение дискретных фрункций, смешанные ряды по полиномам Мейкснера, ортогональным на равномерной сетке.

DOI: 10.18500/1816-9791-2016-16-3-310-321

\section{ВВЕДЕНИЕ}

Теория полиномов, ортогональных относительно скалярных произведений типа Соболева, получила в последние три десятилетия интенсивное развитие и нашла ряд важных приложений (см. [1-6] и цитированную там литературу). Характерной особенностью скалярных произведений типа Соболева 